\title{
A Comparison Between the Nonlocal and the Classical Worlds: Minimal Surfaces, Phase Transitions, and Geometric Flows
}

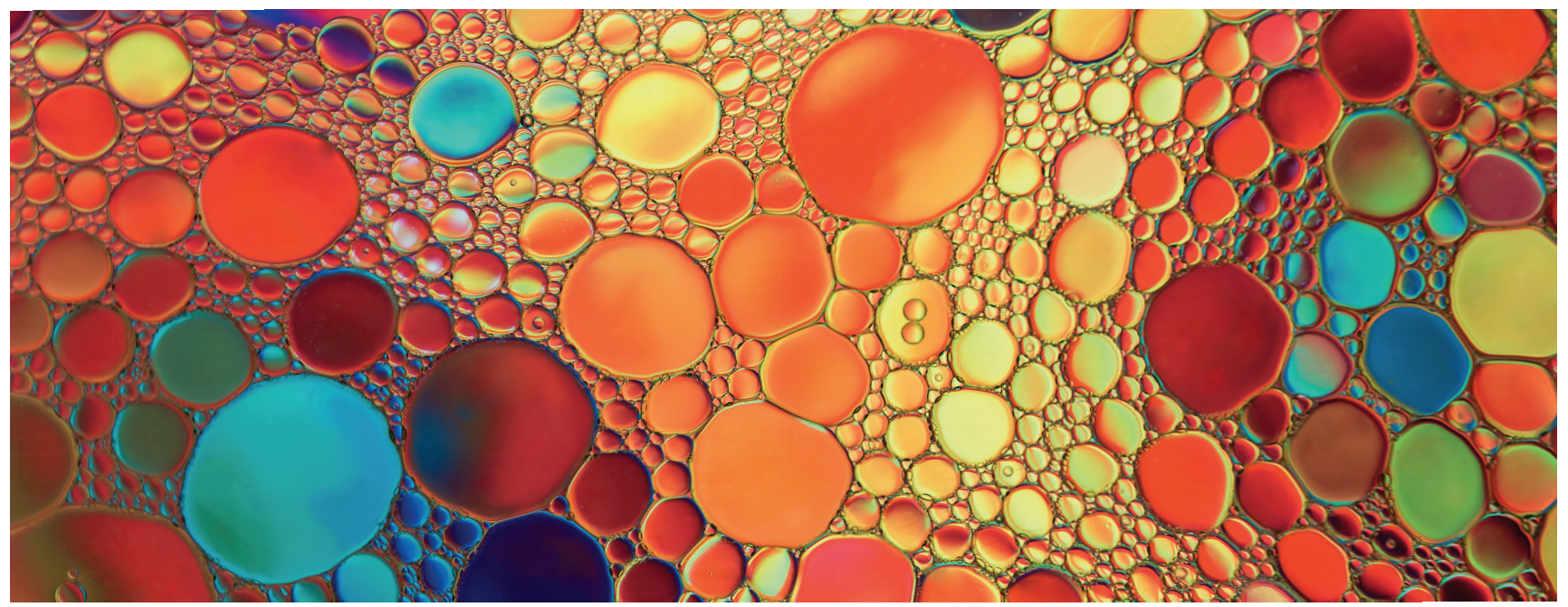

\section{Serena Dipierro}

The nonlocal world presents an abundance of surprises and wonders to discover. These special properties are usually the consequence of long-range interactions, which, especially in the presence of geometric structures and nonlinear phenomena, end up producing a variety of novel patterns. We will briefly discuss some of these features, focusing on the case of (non)local minimal surfaces, (non)local phase coexistence models, and (non)local geometric flows.

Serena Dipierro is an associate professor of mathematics and statistics at the University of Western Australia. Her email address is serena.di pi erro@uwa . edu. au.

Communicated by Notices Associate Editor Daniela De Silva.

For permission to reprint this article, please contact:

reprint-permission@ams.org.

DOI: https://doi.org/10.1090/noti2147

\section{Minimal Surfaces}

1.1. Classical minimal surfaces. The notion of perimeter is one of the concepts we (think we are) most familiar with, and we have been exposed to it since primary school. In spite of its (apparently) elementary nature, understanding perimeter, as well as objects that minimize the perimeter, is an extremely difficult task, involving several advanced methodologies, including a tailor-made definition of perimeter introduced by R. Caccioppoli and relying on the total variation of a step function in the sense of $\mathrm{L}$. Tonelli (as extended by L. Cesari in higher dimensions).

One of the advantages of Caccioppoli's approach to perimeter is its lower semicontinuity, a feature which is not enjoyed by the codimension 1 Hausdorff measure, as we now briefly discuss. Indeed, if $n \geq 2,[0,1]^{n} \cap \mathbb{Q}^{n}=\left\{q_{j}\right\}_{j \in \mathbb{N}}$, and

$$
E_{k}:=\bigcup_{j=1}^{k} B_{\varepsilon / 2^{j}}\left(q_{j}\right),
$$


with $\varepsilon \in(0,1)$ to be conveniently chosen, one sees that $E_{k}$ converges, in $L^{1}\left(\mathbb{R}^{n}\right)$, to the set

$$
E:=\bigcup_{j=1}^{+\infty} B_{\varepsilon / 2^{j}}\left(q_{j}\right)
$$

and that

$$
\begin{aligned}
\mathcal{H}^{n-1}\left(\partial E_{k}\right) & \leq \sum_{j=1}^{k} \mathcal{H}^{n-1}\left(B_{\varepsilon / 2^{j}}\left(q_{j}\right)\right) \\
& \leq \sum_{j=1}^{+\infty} \frac{\mathrm{const}}{2^{j(n-1)}}
\end{aligned}
$$

which is bounded uniformly in $k$ (and also in $\varepsilon$ ). Nevertheless, since $E \supseteq[0,1]^{n} \cap \mathbb{Q}^{n}$, it follows that $\bar{E} \supseteq[0,1]^{n}$, and consequently the $n$-dimensional Lebesgue measure of $\bar{E}$ is bounded from below by 1 , i.e., $\mathcal{H}^{n}(\bar{E}) \geq 1$, up to normalizing constants. On the other hand,

$$
\begin{aligned}
\mathcal{H}^{n}(E) & \leq \sum_{j=1}^{+\infty} \mathcal{H}^{n}\left(B_{\varepsilon / 2^{j}}\left(q_{j}\right)\right) \\
& \leq \sum_{j=1}^{+\infty} \frac{\operatorname{const} \varepsilon^{n}}{2^{j n}} \leq \operatorname{const} \varepsilon^{n},
\end{aligned}
$$

whence

$$
\mathcal{H}^{n}(\partial E) \geq \mathcal{H}^{n}(\bar{E})-\mathcal{H}^{n}(E) \geq \frac{1}{2},
$$

as long as $\varepsilon$ is small enough, and therefore $\mathcal{H}^{n-1}(\partial E)=$ $+\infty$.

This observation and (1) entail that the codimension 1 Hausdorff measure is not lower semicontinuous, making it somewhat unfit for the variational problems related to the perimeter.

In spite of Caccioppoli's innovative approach, the new theory of perimeters offered a number of snares and pitfalls, and several experts maintained a prudent skepticism about this new direction of investigation. In particular, the reviews on Caccioppoli's work by L. C. Young clearly manifested that some statement "can only lead one to doubt its depth, but, in any case, it is difficult, without more details, to assess the scope and generality of a theory presented so sketchily," in which, ultimately, "the reviewer regretfully finds again no more than a misleading plausibility."

Caccioppoli's approach was then fleshed out and exploited to the best of its possibilities by E. De Giorgi, who, after the death of Caccioppoli, christened the sets of finite perimeter "Caccioppoli sets." Interestingly, Young's review of De Giorgi's work appreciated that it "makes it possible to judge more clearly the precise scope of Caccioppoli's definitions."

Interestingly, the point of view of De Giorgi was somewhat conceptually different, though formally equivalent, to that of Caccioppoli. Indeed, Caccioppoli's original definition for the perimeter of a set $E$ relied on a "distributional idea," that is, testing the characteristic function of $E$ against the divergence of all possible vector fields with prescribed $L^{\infty}$-norm, according to the formula

$$
\operatorname{Per}(E, \Omega):=\sup _{\substack{\phi \in C_{0}^{1}(\Omega, \mathbb{R} n) \\\|\phi\|_{L^{\infty}(\Omega)^{\leq 1}}}} \int_{\Omega} \chi_{E}(x) \operatorname{div} \phi(x) d x .
$$

The finiteness of this quantity implies the existence of a vector-valued Radon measure, which is formally the gradient of the characteristic function of $E$, and which allows one to write a suitable "integration by parts formula" of Gauß-Green type (conversely, for smooth sets $E$, a classical integration by parts allows one to identify the quantity in (2) with basically any reasonable notion of surface measure of $\partial E$ ).

De Giorgi's original definition was instead based on a "heat flow" approach. Roughly speaking, one considers the heat flow of the characteristic function of a set $E$ (i.e., its convolution with respect to the usual Gauß kernel), and reconstructs the perimeter in the limit out of the size of the gradient of this caloric function. More explicitly, De Giorgi's definition of perimeter consists of

$$
\operatorname{Per}(E, \Omega):=\lim _{t \searrow 0} \int_{\Omega}\left|D \Phi_{E}(x, t)\right| d x,
$$

where

$$
\Phi_{E}(x, t):=\frac{1}{(\pi t)^{\frac{n}{2}}} \int_{\mathbb{R}^{n}} \chi_{E}(y) e^{-\frac{|x-y|^{2}}{t}} d y,
$$

and this setting indeed coincides with the one in (2), as proved by De Giorgi himself.

1.2. Nonlocal minimal surfaces. In [CRS10], a new notion of nonlocal perimeter has been introduced, and the study of the corresponding minimizers has started. The simple but deep idea grounding this new definition consists of considering pointwise interactions between disjoint sets, modulated by a kernel. The prototype of these interactions considers kernels which have translational, rotational, and dilation invariance. Concretely, given $\sigma \in$ $(0,1)$, one considers the $\sigma$-interaction between two disjoint sets $F$ and $G$ in $\mathbb{R}^{n}$ as defined by

$$
\mathcal{J}_{\sigma}(F, G):=\iint_{F \times G} \frac{d x d y}{|x-y|^{n+\sigma}},
$$

and the $\sigma$-perimeter of a set $E$ in $\mathbb{R}^{n}$ as the $\sigma$-interaction between $E$ and its complement $E^{c}$; namely,

$$
\operatorname{Per}_{\sigma}\left(E, \mathbb{R}^{n}\right):=\mathcal{J}_{\sigma}\left(E, E^{c}\right) .
$$

To deal with local minimizers, given a domain $\Omega \subset \mathbb{R}^{n}$ (say, with sufficiently smooth boundary), it is also convenient to introduce the notion of $\sigma$-perimeter of a set $E$ in $\Omega$. To this end, one can consider the interaction in (4) 


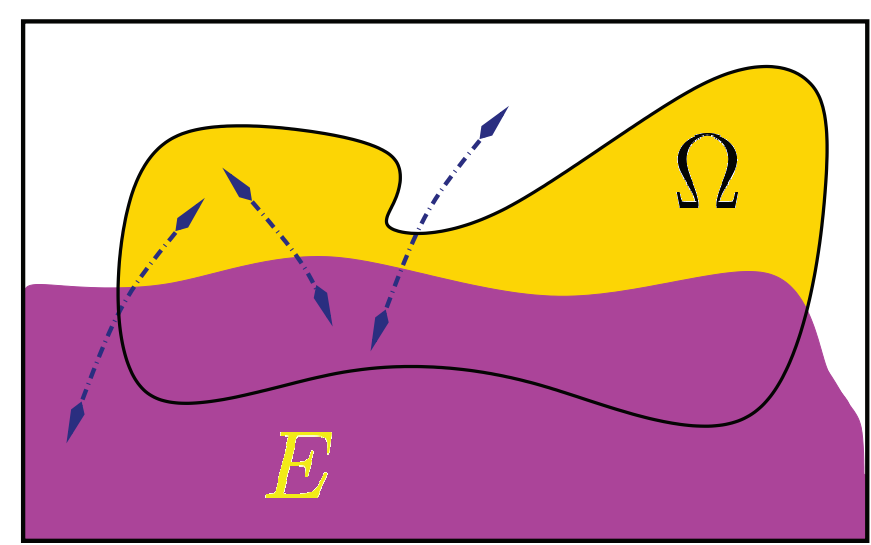

Figure 1. Long-range interactions leading to the fractional perimeter of the set $E$ in $\Omega$.

as composed by four different terms (by considering the intersections of $E$ and $E^{c}$ with $\Omega$ and $\Omega^{c}$ ); namely, we can rewrite (4) as

$$
\begin{aligned}
& \operatorname{Per}_{\sigma}\left(E, \mathbb{R}^{n}\right)=\mathcal{J}_{\sigma}\left(E \cap \Omega, E^{c} \cap \Omega\right) \\
& \quad+\mathcal{J}_{\sigma}\left(E \cap \Omega, E^{c} \cap \Omega^{c}\right)+\mathcal{J}_{\sigma}\left(E \cap \Omega^{c}, E^{c} \cap \Omega\right) \\
& \quad+\mathcal{J}_{\sigma}\left(E \cap \Omega^{c}, E^{c} \cap \Omega^{c}\right) .
\end{aligned}
$$

Among the four terms in the right-hand side of (5), the first three terms take into account interactions in which at least one contribution comes from $\Omega$ (specifically, all the contributions to $\mathcal{J}_{\sigma}\left(E \cap \Omega, E^{c} \cap \Omega\right)$ come from $\Omega$, while the contributions to $\mathcal{J}_{\sigma}\left(E \cap \Omega, E^{c} \cap \Omega^{c}\right)$ and $\mathcal{J}_{\sigma}\left(E \cap \Omega^{c}, E^{c} \cap \Omega\right)$ come from the interactions of points in $\Omega$ with points in $\Omega^{c}$ ). The last term in (5) is structurally different, since it only takes into account contributions coming from outside $\Omega$. It is therefore natural to define the $\sigma$-perimeter of a set $E$ in $\Omega$ as the collection of all the contributions in (5) that take into account points in $\Omega$, thus defining

$$
\begin{aligned}
& \operatorname{Per}_{\sigma}(E, \Omega):=\mathcal{J}_{\sigma}\left(E \cap \Omega, E^{c} \cap \Omega\right) \\
& \quad+\mathcal{J}_{\sigma}\left(E \cap \Omega, E^{c} \cap \Omega^{c}\right)+\mathcal{J}_{\sigma}\left(E \cap \Omega^{c}, E^{c} \cap \Omega\right) .
\end{aligned}
$$

See Figure 1.

This notion of perimeter recovers the classical one as $\sigma \nearrow 1$ in various senses, and indeed the analogy between the minimizers of (6) with respect to prescribed sets outside $\Omega$ (named "nonlocal minimal surfaces" in [CRS10]) and the minimizers of the classical perimeter (the "classical minimal surfaces") has been widely exploited in [CRS10] as well as in a series of subsequent articles, such as [CV13]. Notwithstanding the important similarities between the nonlocal and the classical settings, many striking differences arise, as we will also describe in this note.

Minimizers, and more generally critical points, of the $\sigma$-perimeter satisfy (with a suitable viscosity meaning, and in a principal value sense) the equation

$$
\begin{gathered}
\mathcal{H}_{E}^{\sigma}(x):=\int_{\mathbb{R}^{n}} \frac{\Xi_{E}(y)}{|x-y|^{n+\sigma}} d y=0, \\
\text { for } x \in(\partial E) \cap \Omega,
\end{gathered}
$$

being

$$
\Xi_{E}(y):= \begin{cases}-1 & \text { if } x \in E, \\ 1 & \text { if } x \in E^{c}\end{cases}
$$

see [CRS10]. The quantity $\mathcal{H}_{E}^{\sigma}$ can be seen as a "nonlocal mean curvature," and indeed it approaches in various senses the classical mean curvature $\mathcal{H}_{E}^{1}$. Interestingly, the nonlocal mean curvature measures the size of $E$ with respect to its complement, in an integral fashion weighted by the interaction kernel, in a precise form given by (7). This interpretation is indeed closely related with the classical mean curvature, in which the size of $E$ is measured only "in the vicinity of the boundary," since (up to normalizing constants)

$$
\mathcal{H}_{E}^{1}(x)=\lim _{\rho \searrow 0} \frac{1}{\rho^{n+1}} \int_{B_{\rho}(x)} \Xi_{E}(y) d y \quad \text { for } x \in \partial E .
$$

The settings in (4) and (7) also reveal the "fractional notion" of these nonlocal minimal surfaces. As a matter of fact, if one considers the fractional Gagliardo seminorm defined, for every $s \in(0,1)$, by

$$
[f]_{H^{s\left(\mathbb{R}^{n}\right)}}:=\sqrt{\iint_{\mathbb{R}^{n} \times \mathbb{R}^{n}} \frac{|f(x)-f(y)|^{2}}{|x-y|^{n+2 s}} d x d y,}
$$

it follows that $\left[\Xi_{E}\right]_{H^{\sigma / 2}\left(\mathbb{R}^{n}\right)}^{2}=8 \operatorname{Per}_{\sigma}(E)$.

Moreover, defining the fractional Laplacian as

$$
(-\Delta)^{s} f(x):=\int_{\mathbb{R}^{n}} \frac{f(x)-f(y)}{|x-y|^{n+2 s}} d y,
$$

and, for a smooth set $E$, identifying a.e. the function $\Xi_{E}$ with

$$
\widetilde{\Xi}_{E}(y):= \begin{cases}-1 & \text { if } x \text { is in the interior of } E, \\ 1 & \text { if } x \text { is in the interior of } E^{c}, \\ 0 & \text { if } x \in \partial E,\end{cases}
$$

one sees that, for $x \in \partial E$,

$$
\begin{aligned}
\mathcal{H}_{E}^{\sigma}(x) & =\int_{\mathbb{R}^{n}} \frac{\widetilde{\Xi}_{E}(y)}{|x-y|^{n+\sigma}} d y \\
& =\int_{\mathbb{R}^{n}} \frac{\widetilde{\Xi}_{E}(y)-\widetilde{\Xi}_{E}(x)}{|x-y|^{n+\sigma}} d y \\
& =-(-\Delta)^{\sigma / 2} \widetilde{\Xi}_{E}(x) .
\end{aligned}
$$

In this sense, one can relate "geometric" equations, such as (7), with "linear" equations driven by the fractional Laplacian (as in (8)), in which, however, the nonlinear feature of the problem is encoded by the fact that the equation takes place on the boundary of a set (once again, however, sharp differences arise between nonlocal minimal surfaces 
and solutions of linear equations, as we will discuss in the rest of this note).

From the considerations above, we see how the study of nonlocal minimal surfaces is therefore related to the one of hypersurfaces with vanishing nonlocal mean curvature, and a direction of research lies in finding correspondences between critical points of nonlocal and classical perimeters. With respect to this point, we recall that double helicoids possess both vanishing classical mean curvature and vanishing nonlocal mean curvature, as noticed in [CDDP16]. Also, nonlocal catenoids have been constructed in [DdPW18] by bifurcation methods from the classical case. Differently from the local situation, the nonlocal catenoids exhibit linear growth at infinity, rather than a logarithmic one, as recently established by M. Cozzi and E. Valdinoci.

The cases of hypersurfaces with prescribed nonlocal mean curvature and of minimizers of nonlocal perimeter functionals under periodic conditions have also been considered in the literature. We also mention that we are restricting here to the notion of nonlocal minimal surfaces of fractional type, as introduced in [CRS10], but other very interesting, and structurally different, notions of nonlocal minimal surfaces have also been considered in the literature.

\section{Interior Regularity for Minimal Surfaces}

A first step to understanding the geometric properties of (both classical and nonlocal) minimal surfaces is to detect their regularity properties and the possible singularities. While the regularity theory of classical minimal surfaces is a rather well-established topic, many basic regularity problems in the nonlocal minimal surfaces setting are open, and they require brand new ideas to be attacked.

2.1. Interior regularity for classical minimal surfaces. Classical minimal surfaces are smooth up to dimension 7. This was established when $n=3$ by E. De Giorgi in 1965, when $n=4$ by F. J. Almgren in 1966, and when $n \leq 7$ by J. Simons in 1968.

Also, it was conjectured by J. Simons that this regularity result was optimal in dimension 7 , suggesting as a possible counterexample in dimension 8 the cone

$$
\left\{(x, y) \in \mathbb{R}^{4} \times \mathbb{R}^{4} \text { s.t. }|x|<|y|\right\} .
$$

This conjecture was indeed positively assessed by $\mathrm{E}$. Bombieri, E. De Giorgi, and E. Giusti in 1969, thus showing that classical minimal surfaces can develop singularities in dimension 8 and higher.

Besides the smoothness, the analyticity of minimal surfaces in dimension up to 7 was established by E. De Giorgi in 1957.

2.2. Interior regularity for nonlocal minimal surfaces. Different from the classical case, the regularity theory for nonlocal minimal surfaces is still mostly open. Until now, a complete result holds only in dimension 2 , since the smoothness of nonlocal minimal surfaces was established in [SV13].

In higher dimensions, the results in [CV13] give that nonlocal minimal surfaces are smooth up to dimension 7 as long as $\sigma$ is sufficiently close to 1 : namely, for every $n \in \mathbb{N} \cap[1,7]$ there exists $\sigma(n) \in[0,1)$ such that all $\sigma$-nonlocal minimal surfaces in dimension $n$ are smooth provided that $\sigma \in(\sigma(n), 1)$. The optimal value of $\sigma(n)$ is unknown (except when $n=1,2$, in which case $\sigma(n)=0$ ).

This result strongly relies on the fact that the nonlocal perimeter approaches the classical perimeter when $\sigma \nearrow 1$, and therefore one can expect that when $\sigma$ is close to 1 nonlocal minimal surfaces inherit the regularity of classical minimal surfaces.

On the other hand, we remark that it is not possible to obtain information on the regularity of nonlocal minimal surfaces from the asymptotics of the fractional perimeter as $\sigma \searrow 0$. Indeed, it has been proved in [DFPV13] that the fractional perimeter converges, roughly speaking, to the Lebesgue measure of the set when $\sigma \searrow 0$, and so in this case minimizers can be as wild as they wish.

As a matter of fact, the smoothness obtained in [SV13] and [CV13] is of $C^{1, \alpha}$-type; the improvement to $C^{\infty}$ was obtained in [BFV14].

Indeed, nonlocal minimal surfaces enjoy an "improvement of regularity" from locally Lipschitz to $C^{\infty}$ (more precisely, from locally Lipschitz to $C^{1, \alpha}$ for any $\alpha<\sigma$, thanks to [FV17], and from $C^{1, \alpha}$ for some $\alpha>\sigma / 2$ to $C^{\infty}$, thanks to [BFV14]).

It is an open problem to establish whether smooth nonlocal minimal surfaces are actually analytic. It is also open to determine whether or not singular nonlocal minimal surfaces exist (a preliminary analysis performed in [DdPW18] for symmetric cones when the fractional parameter $\sigma$ approaches 0 may lead to the conjecture that nonlocal minimal surfaces are smooth up to dimension 6 , but completely new phenomena may arise in dimension 7 when $\sigma$ is close to 0 ).

Quantitative versions of the regularity results for nonlocal minimal surfaces have been obtained in [CSV19].

As a matter of fact, in [CSV19] more general interaction kernels have also been considered, and regularity results have been obtained for more general critical points than minimizers. In particular, one can consider stable surfaces with vanishing nonlocal mean curvature as critical points with "nonnegative second variations" of the functional. More precisely, one says that $E$ is "stable" for the nonlocal perimeter in $\Omega$ if $\operatorname{Per}_{\sigma}(E, \Omega)<+\infty$ and for every vector field $X=X(x, t) \in C^{2}\left(\mathbb{R}^{n} \times(-1,1) ; \mathbb{R}^{n}\right)$, which is compactly supported in $\Omega$ and whose integral flow is denoted by $\Phi_{X}^{t}$, and for every $\varepsilon>0$, there exists $t_{0}>0$ such 
that

$$
\text { and } \begin{array}{r}
\operatorname{Per}_{\sigma}\left(\Phi_{X}^{t}(E) \cup E\right)-\operatorname{Per}_{\sigma}(E)+\varepsilon t^{2} \geq 0 \\
\left.\operatorname{Per}_{X}^{t}(E) \cap E\right)-\operatorname{Per}_{\sigma}(E)+\varepsilon t^{2} \geq 0
\end{array}
$$

for all $t \in\left(-t_{0}, t_{0}\right)$.

Interestingly, the notion of stability in the nonlocal regime provides stronger information with respect to the classical counterpart: for instance, if $E$ is stable for the nonlocal perimeter in $B_{2 R}$, then

$$
\operatorname{Per}\left(E, B_{R}\right) \leq C R^{n-1},
$$

where $C>0$ is a constant depending only on $n$ and $\sigma$.

We stress that the left-hand side in (9) involves the classical perimeter (not the nonlocal perimeter); hence an estimate of this type is quite informative also for minimizers (not only for stable sets). In a sense, up to now, the perimeter estimate in (9) is the only regularity result known for nonlocal minimal surfaces (and, more generally, for nonlocal stable surfaces) in any dimension (differently from [SV13] and [CV13], this estimate does not imply the smoothness of the surface, but only a bound on the perimeter).

Moreover, the right-hand side of (9) is uniform with respect to the external data of $E$. In particular, wild data are shown to have a possible impact on the perimeter of $E$ near the boundary, but not in the interior (for instance, nonlocal minimal surfaces and, more generally, nonlocal stable surfaces in $B_{2}$ have a uniformly bounded perimeter in $B_{1}$ ). This is a remarkable property, heavily relying on the nonlocal structure of the problem, which has no counterpart in the classical case. As an example, one can consider a family of parallel hyperplanes, which is a local minimizer for the perimeter: since each hyperplane produces a certain perimeter contribution in $B_{1}$, no uniform bound can be attained in this situation. In this sense, a bound as in (9) prevents arbitrary families of possibly perturbed hyperplanes to be stable surfaces for the nonlocal perimeter.

Concerning the regularity of stable sets for the nonlocal perimeter, we also mention that half-spaces are the only stable cones in $\mathbb{R}^{3}$ if the fractional parameter $\sigma$ is sufficiently close to 1 , as recently proved by X. Cabré, E. Cinti, and J. Serra.

\section{The Dirichlet Problem for Minimal Surfaces}

Another classical problem in the theory of minimal surfaces is finding solutions with graphical structure for given boundary or exterior data. In the classical setting, this corresponds to studying graphs with vanishing mean curvature inside a given domain with a prescribed Dirichlet datum along the boundary of the domain. Its nonlocal counterpart consists of studying graphs with vanishing nonlocal mean curvature inside a given domain with a prescribed datum outside this domain. We discuss now similarities and differences between these two problems.
3.1. The Dirichlet problem for classical minimal surfaces. A classical problem in geometric analysis is to seek hypersurfaces with vanishing mean curvature and prescribed boundary data. Namely, given a smooth domain $\Omega \subset \mathbb{R}^{n}$ and a boundary datum $\varphi \in C(\partial \Omega)$, the problem is to find $u \in C^{2}(\Omega) \cap C(\bar{\Omega})$ that solves the Dirichlet problem

$$
\begin{cases}\operatorname{div}\left(\frac{\nabla u}{\sqrt{1+|\nabla u|^{2}}}\right)=0 & \text { in } \Omega, \\ u=\varphi & \text { on } \partial \Omega .\end{cases}
$$

A classical approach to this problem consists of fixing $M>$ 0 and using the Ascoli theorem to minimize the area functional among functions in

$$
\begin{array}{r}
X_{M}:=\left\{u \in C^{0,1}(\bar{\Omega}) \text { with } u=\varphi \text { on } \partial \Omega\right. \\
\text { and } \left.[u]_{C^{0,1}(\bar{\Omega})} \leq M\right\},
\end{array}
$$

where, as customary, we denote the Lipschitz seminorm of $u$ by

$$
[u]_{C^{0,1}(\bar{\Omega})}:=\sup _{\substack{x, y \in \Omega \\ x \neq y}} \frac{|u(x)-u(y)|}{|x-y|} .
$$

To use this direct minimization approach, one needs of course to check that $X_{M} \neq \emptyset$. Furthermore, in order to obtain a solution to (10), it is crucial that the Lipschitz seminorm of the minimizer in $X_{M}$ is in fact strictly smaller than $M$ (as long as $M$ is chosen conveniently large), so as to obtain an interior minimum of the area functional, and thus find (10) as the Euler-Lagrange equation of this minimization procedure (the Lipschitz bound permitting the use of uniformly elliptic regularity theory for PDEs, leading to the desired smoothness of the solution inside the domain).

Finding sufficient and necessary conditions for this procedure to work and, in general, for obtaining solutions to (10) has been a classical topic of investigation. The main lines of this research took into account a "bounded slope condition" on the domain and the datum that allows one to exploit affine functions as barriers. In this, the convexity of $\Omega$ played an important role for the explicit construction of linear barriers.

The optimal conditions for existence results were discovered by H. Jenkins and J. Serrin in 1968 and rely on the notion of "mean convexity" of the domain: namely, if $\Omega$ has $C^{2}$ boundary, problem (10) is solvable for every continuous boundary datum $\varphi$ if and only if the mean curvature of $\Omega$ is nonnegative (when $n=2$, the notion of mean convexity boils down to the usual convexity).

3.2. The Dirichlet problem for nonlocal minimal surfaces. Given a smooth domain $\Omega \subset \mathbb{R}^{n}$, one considers the cylinder $\Omega^{\star}:=\Omega \times \mathbb{R}$ and looks for local minimizers of the nonlocal perimeter among all the sets with prescribed datum outside $\Omega^{\star}$ (more precisely, one seeks local minimizers of the nonlocal perimeter in $\widetilde{\Omega}$ for every smooth 


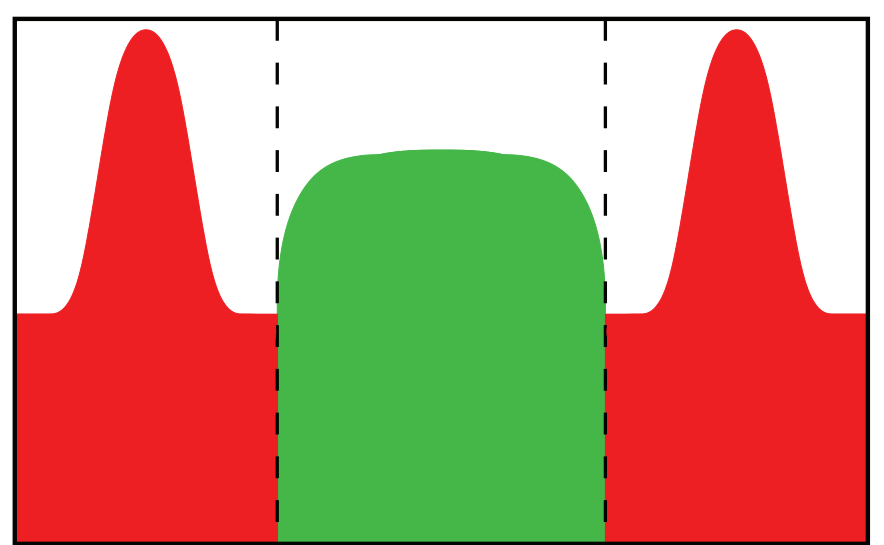

Figure 2. An example of stickiness (the red is the prescribed set, the green is the $s$-minimizer).

and bounded $\widetilde{\Omega} \subset \Omega^{\star}$; see the PhD thesis of L. Lombardini for all the details of this construction).

In [DSV16], it is shown that this problem is solvable in the class of graphs. More precisely, if $E$ is a local minimizer of the $\sigma$-perimeter in $\Omega^{\star}$ such that its datum outside $\Omega^{\star}$ has a graphical structure, namely,

$$
E \backslash \Omega^{\star}=\left\{x_{n+1}<u_{0}(x), x \in \mathbb{R}^{n} \backslash \Omega\right\},
$$

for some continuous function $u_{0}: \mathbb{R}^{n} \rightarrow \mathbb{R}$, then $E$ has a graphical structure inside $\Omega^{\star}$ as well, that is,

$$
E \cap \Omega^{\star}=\left\{x_{n+1}<u(x), x \in \Omega\right\}
$$

for some continuous function $u: \mathbb{R}^{n} \rightarrow \mathbb{R}$.

Remarkably, in general this problem may lose continuity at the boundary of $\Omega^{\star}$. That is, in the setting of (11) and (12), it may happen that

$$
\lim _{\substack{x \rightarrow \partial \Omega \\ x \in \Omega}} u(x) \neq \lim _{\substack{x \rightarrow \partial \Omega \\ x \in \Omega^{c}}} u_{0}(x) .
$$

The first example of this quite surprising phenomenon was given in [DSV17], and this is indeed part of a general and remarkable structure of nonlocal minimal surfaces that we named "stickiness": namely, nonlocal minimal surfaces have the tendency to stick at the boundary of the domain (even when the domain is convex, in sharp contrast with the pattern exhibited by classical minimal surfaces). See, e.g., Figures 2 and 3 for some qualitative examples.

Recently, in [DSV20], it was discovered that the boundary discontinuity pointed out in (13) is indeed a "generic" phenomenon at least in the plane: namely, if a given graphical external datum produces a continuous minimizer, then an arbitrarily small perturbation of it will produce a minimizer exhibiting the boundary discontinuity.

Interestingly, one of the main ingredients in the proof of the genericity of the boundary discontinuity in [DSV20] is an "enhanced boundary regularity": namely, nonlocal minimal surfaces in the plane with a graphical structure that are continuous at the boundary are automatically

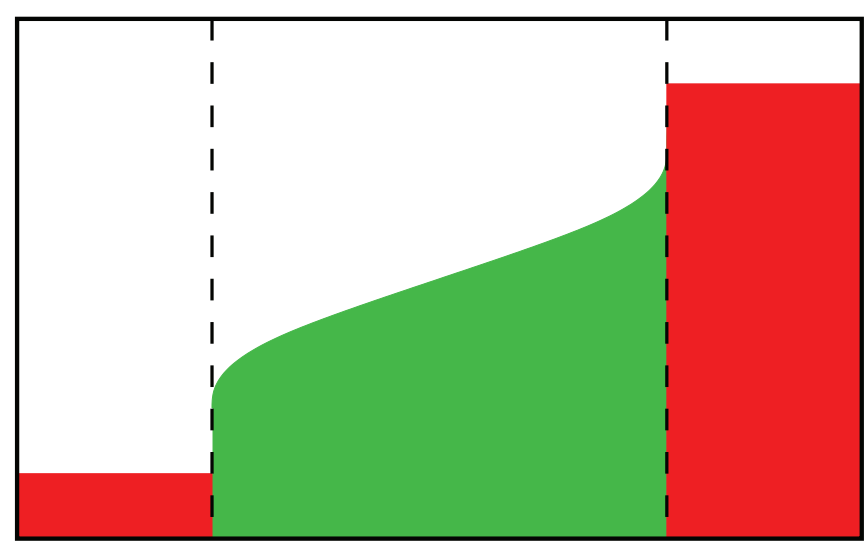

Figure 3. Another example of stickiness (the red is the prescribed set, the green is the $s$-minimizer).

$C^{1, \frac{1+\sigma}{2}}$ at the boundary (that is, boundary continuity implies boundary differentiability).

A byproduct of this construction, taking into account also the boundary properties obtained in [CDSS16], is also that nonlocal minimal surfaces with a graphical structure in the plane exhibit a "butterfly effect" for their boundary derivatives: namely, while a nonlocal minimal graph that is continuous at the boundary has also a finite derivative there, an arbitrarily small perturbation of the exterior graph will produce not only a jump at the boundary but also an infinite boundary derivative (that is, the boundary derivative switches from a finite, possibly zero, value to infinity only due to an arbitrarily small perturbation of the datum).

It is worth recalling that the boundary discontinuity of nonlocal minimal surfaces is not only a special feature of the nonlocal world, when compared to the case of classical minimal surfaces, but also a special feature of the nonlinear equation of nonlocal mean curvature type, when compared to the case of linear fractional equations: indeed, as detailed in [ROS14], solutions of linear equations of the type $(-\Delta)^{s} u=f$ in a domain $\Omega$ with an external datum are typically Hölder continuous up to the boundary of $\Omega$, and no better than this (instead, nonlocal minimal surfaces are typically discontinuous at the boundary, but when they are continuous they are also differentiable).

Though this discrepancy between nonlocal minimal surfaces and solutions of linear equations seems a bit surprising at first glance, a possible explanation for it lies in the "accuracy" of the approximation that a linear equation can offer to an equation of geometric type, such as the one related to the nonlocal mean curvature. Namely, linear equations end up being a good approximation of the geometric equation with respect to the normal of the surface itself: hence, when the surface is "almost horizontal" the graphical structure of the geometric equation is well shadowed by its linear counterpart, but when the surface is 
"almost vertical" the linear counterpart of the geometric equation should take into account not the graph of the original surface but its "inverse" (i.e., the graph describing the surface in the horizontal, rather than vertical, direction).

We also refer to [DSV] for a first analysis of the stickiness phenomenon in dimension 3.

Many examples of nonlocal minimal surfaces exhibiting the stickiness phenomenon are studied in detail in a 2019 article by C. Bucur, L. Lombardini, and E. Valdinoci, also with respect to a suitable parameter measuring the weighted measure of the datum at infinity.

We also remark that nonlocal minimal surfaces with a graphical structure enjoy special regularity properties: for instance, they are smooth inside their domain of minimization, as established in [CC19].

\section{Growth and Bernšteĭn Properties of Minimal Graphs}

A classical question, dating back to the 1927 article by S. Bernštern, is whether or not minimizers of a given geometric problem which have a global graphical structure are necessarily hyperplanes. If this feature holds, we say that the problem enjoys the "Bernšteinn property."

This question is also related to the growth of these minimal graphs at infinity. We now discuss and compare the classical and the nonlocal worlds with respect to these features.

4.1. Growth and Bernšteinn properties of classical minimal graphs. The Bernštern problem for classical minimal surfaces asks whether or not solutions of

$$
\operatorname{div}\left(\frac{\nabla u}{\sqrt{1+|\nabla u|^{2}}}\right)=0 \text { in } \mathbb{R}^{n}
$$

are necessarily affine functions.

When $n=2$, a positive answer to this question was provided by S. Bernštein in 1927. The development of the theory related to the classical Bernštein problem is closely linked to the regularity theory of classical minimal surfaces, since, as proved by E. De Giorgi in 1965, the falsity of the Bernšteĭn property in $\mathbb{R}^{n}$ would imply the existence of a singular minimal surface in $\mathbb{R}^{n}$.

In light of this connection, the Bernštein property for classical minimal surfaces was established by E. De Giorgi in 1965 for $n=3$, by F. J. Almgren in 1966 for $n=4$, and by J. Simons in 1968 up to $n=7$ (compare with the references in section 2.1). A counterexample when $n=8$ was then provided by E. Bombieri, E. De Giorgi, and E. Giusti in 1969, by constructing an eight-dimensional graph in $\mathbb{R}^{9}$ which has vanishing mean curvature without being affine.

As a counterpart of this kind of problem, a precise growth analysis of classical minimal surfaces with graphical structure was obtained by R. Finn in 1963 when $n=2$ and by E. Bombieri, E. De Giorgi, and M. Miranda in 1969 for all dimensions.

More precisely, if $u$ is a solution of

$$
\operatorname{div}\left(\frac{\nabla u}{\sqrt{1+|\nabla u|^{2}}}\right)=0 \text { in } B_{R} \subset \mathbb{R}^{n},
$$

then

$$
\sup _{B_{R}}|\nabla u| \leq \exp \left[C\left(1+\frac{\sup _{2 R} u-u(0)}{R}\right)\right]
$$

for some $C>0$ depending only on $n$. Interestingly, this exponential type of gradient estimate is optimal, as demonstrated by R. Finn in 1963.

4.2. Growth and Bernštein properties of nonlocal minimal graphs. The Bernštein properties of nonlocal minimal graphs have been investigated in [FV17]. In particular, it is proved in these works that the falsity of the nonlocal Bernštein property in $\mathbb{R}^{n}$ would imply the existence of a singular nonlocal minimal surface in $\mathbb{R}^{n}$, thus providing a perfect counterpart of the 1965 result by De Giorgi to the nonlocal setting.

Combining this with the regularity results for nonlocal minimal surfaces in [SV13] and [CV13], one obtains that the Bernšteĭn property holds true for solutions $u: \mathbb{R}^{n} \rightarrow$ $\mathbb{R}$ of the $\sigma$-mean curvature equation in (7) provided that either $n \in\{1,2\}$, or $n \leq 7$ and $\sigma$ is sufficiently close to 1 .

It is an open problem to prove or disprove the Bernštein property for nonlocal minimal graphs $u: \mathbb{R}^{n} \rightarrow \mathbb{R}$ in the general case when $n>2$ and $\sigma \in(0,1)$ (the analysis on symmetric cones performed in [DdPW18] might suggest that new phenomena could arise in dimension 7 when the parameter $\sigma$ is close to 0 ).

Concerning the growth of nonlocal minimal surfaces, it has been established in [CC19] that if $u: B_{2 R} \subset \mathbb{R}^{n} \rightarrow \mathbb{R}$ is a solution of the $\sigma$-mean curvature equation in $B_{2 R}$, then

$$
\sup _{B_{R}}|\nabla u| \leq C\left(1+\frac{\sup _{R} u-\inf u}{B_{R}}\right)^{n+1+\sigma}
$$

for a suitable constant $C>0$, depending only on $n$ and $\sigma$.

On the one hand, comparing (14) and (15), we observe that the nonlocal world offers us a wealth of surprises: first of all, the estimate in (15) is of polynomial type, rather than of exponential type, as was the bound in (14). Roughly speaking, this is due to the fact that two different and sufficiently close "ends" of a nonlocal minimal surface repel each other (compare the discussion about the nonlocal catenoid on page 1327). Moreover, the right-hand side of (15) presents the oscillation of the solution in the same ball $B_{R}$, while (14) needed to extend the right-hand side to a larger ball such as $B_{2 R}$. 
On the other hand, the nonlocal world maintains its own special difficulties: for instance, different from the classical case, it is an open problem to determine whether the estimate in (15) is optimal.

See Table 1 for a sketchy description of some similarities and differences between classical and nonlocal minimal surfaces.

\section{Phase Coexistence Models}

A physical situation in which minimal interfaces naturally arise occurs in the mathematical description of phase coexistence (or phase transition). In the classical setting, the ansatz is that the short range particle interaction produces a surface tension that leads, on a large scale, to interfaces of minimal area. A modern variation of this model takes into account long-range particle interactions, and we describe here in which sense this model is related to the theory of (non)local minimal surfaces.

5.1. Classical phase coexistence models. One of the most popular phase coexistence models is the one producing the so-called Allen-Cahn equation

$$
-\Delta u=u-u^{3} .
$$

Equation (16) has a variational structure, corresponding to critical points of an energy functional of the type

$$
\mathcal{F}(u ; \Omega)=\mathcal{F}(u):=\int_{\Omega}|\nabla u(x)|^{2}+W(u(x)) d x,
$$

where $W$ is a "double-well" potential attaining its minima at the "pure phases" -1 and +1 .

A classical link between the functional in (17) and the perimeter can be expressed in terms of $\Gamma$-convergence, as stated by L. Modica and S. Mortola in 1977. More precisely, given a smooth domain $\Omega \subset \mathbb{R}^{n}$ and $\varepsilon>0$, one can consider the rescaled functional

$$
\mathcal{F}_{\varepsilon}(u ; \Omega)=\mathcal{F}_{\varepsilon}(u):=\int_{\Omega} \varepsilon|\nabla u(x)|^{2}+\frac{1}{\varepsilon} W(u(x)) d x .
$$

Interestingly, the functional $\mathcal{F}_{\varepsilon}$ in (18) inherits the minimization properties of the functional $\mathcal{F}$ in (17) after a dilation: for instance, if $u$ is a minimizer for $\mathcal{F}$ in every ball, then

$$
u_{\varepsilon}(x):=u\left(\frac{x}{\varepsilon}\right)
$$

is a minimizer for $\mathcal{F}_{\varepsilon}$ in every ball. Moreover, it holds that:

- for any $u_{\varepsilon}: \mathbb{R}^{n} \rightarrow[-1,1]$ converging to $u$ in $L_{\text {loc }}^{1}\left(\mathbb{R}^{n}\right)$, we have that

$$
\liminf _{\varepsilon \searrow 0} \mathcal{F}_{\varepsilon}\left(u_{\varepsilon}\right) \geq \mathcal{F}_{0}(u)
$$

- given $u: \mathbb{R}^{n} \rightarrow[-1,1]$, there exists $u_{\varepsilon}: \mathbb{R}^{n} \rightarrow$ $[-1,1]$ converging to $u$ in $L_{\text {loc }}^{1}\left(\mathbb{R}^{n}\right)$, such that

$$
\limsup _{\varepsilon \backslash 0} \mathcal{F}_{\varepsilon}\left(u_{\varepsilon}\right) \leq \mathcal{F}_{0}(u),
$$

where, for a suitable $c>0$,

$$
\mathcal{F}_{0}(u ; \Omega)=\mathcal{F}_{0}(u):= \begin{cases}c \operatorname{Per}(E, \Omega) & \text { if } u=\Xi_{E} \\ & \text { for some set } E, \\ +\infty & \text { otherwise. }\end{cases}
$$

This type of "functional convergence" also has a "geometric counterpart," given by the locally uniform convergence of levels sets, as established by L. A. Caffarelli and A. Córdoba in 1995. More precisely, if $u$ is a minimizer for $\mathcal{F}$ in every ball and $u_{\varepsilon}$ is as in (19), then $u_{\varepsilon}$ converges, up to subsequences, in $L_{\text {loc }}^{1}\left(\mathbb{R}^{n}\right)$ to some $u=\Xi_{E}$, with $E$ minimizing the perimeter, and the level sets of $u_{\varepsilon}$ approach $\partial E$ locally uniformly. That is, given any $\vartheta \in(0,1)$, any $R>0$, and any $\delta>0$ there exists $\varepsilon_{0} \in(0,1)$ such that if $\varepsilon \in\left(0, \varepsilon_{0}\right)$, we have that

$$
\left\{\left|u_{\varepsilon}\right|<1-\theta\right\} \cap B_{R} \subseteq \bigcup_{p \in \partial E} B_{\delta}(p) .
$$

The level set convergence in (23) relies on suitable "energy and density estimates" stating that the energy behaves as an "interface" and, in a neighborhood of the interface, both the phases have positive densities. More precisely, if $R \geq 1$ and $u$ is a minimizer of $\mathcal{F}$ in $B_{R+1}$, then

$$
\mathcal{F}_{B_{R}}(u) \leq C R^{n-1},
$$

for some $C>0$, and for every $\vartheta_{1}, \vartheta_{2} \in(0,1)$, if $|u(0)| \leq \vartheta_{1}$, then

$$
\begin{array}{ll} 
& \left\{u \geq \vartheta_{2}\right\} \cap B_{R} \geq c R^{n} \\
\text { and } \quad & \left\{u \leq-\vartheta_{2}\right\} \cap B_{R} \geq c R^{n} .
\end{array}
$$

5.2. Long-range phase coexistence models. In the recent literature a number of models have been introduced in order to study phase coexistence driven by long-range particle interactions. We describe here a simple model of this kind, comparing the results obtained in this situation with the classical ones presented in section 5.1.

We focus on a nonlocal version of the Allen-Cahn equation (16) given by

$$
(-\Delta)^{s} u=u-u^{3}
$$

with $s \in(0,1)$. Equation (26) has a variational structure, corresponding to critical points of an energy functional of the type

$$
\begin{aligned}
\mathcal{E}(u ; \Omega)= & \mathcal{E}(u):=\int_{Q_{\Omega}} \frac{|u(x)-u(y)|^{2}}{|x-y|^{n+2 s}} d x d y \\
& +\int_{\Omega} W(u(x)) d x,
\end{aligned}
$$

where $W$ is a "double-well" potential attaining its minima at the "pure phases" -1 and +1 , and $Q_{\Omega}$ is the cross-type domain given by

$$
(\Omega \times \Omega) \cup\left(\Omega \times \Omega^{c}\right) \cup\left(\Omega^{c} \times \Omega\right) .
$$




\begin{tabular}{|l|l|l|}
\hline & classical minimal surfaces & nonlocal minimal surfaces \\
\hline interior regularity & up to $n=7$ (optimal) & $\begin{array}{l}\text { known when } n=2 \text {, and up } \\
\text { to } n=7 \text { if } \sigma \text { is large enough } \\
\text { (optimality unknown, no sin- } \\
\text { gular example available) }\end{array}$ \\
\hline $\begin{array}{l}\text { boundary regularity } \\
\text { boundary discontinuity (but } \\
\text { enhanced differentiable regu- } \\
\text { larity if continuous) }\end{array}$ & $\begin{array}{l}\text { yes for convex (or mean con- } \\
\text { vex) domains }\end{array}$ & up to $n=7$ (optimal) \\
\hline gradient bound & $\begin{array}{l}\text { known when } n=2, \text { and up to } \\
n=7 \text { if } \sigma \text { is large enough (op- } \\
\text { timality unknown, no coun- } \\
\text { terexample available) }\end{array}$ \\
\hline
\end{tabular}

Table 1. Classical versus nonlocal minimal surfaces.

The rationale behind this choice of $Q_{\Omega}$ is to collect all the couples of points in which at least one of the two lies in $\Omega$, so as to comprise all the point interactions that involve $\Omega$ (a similar choice was performed in the geometric problem (6)).

The $\Gamma$-convergence theory for the functional in (27) has been established in [SV12]. For this, one needs to introduce a family of rescaled functionals with different scaling properties depending on the fractional parameter $s$. More precisely, it is convenient to define

$$
\begin{aligned}
\mathcal{E}_{\varepsilon}(u ; \Omega)= & \mathcal{E}_{\varepsilon}(u):=a_{\varepsilon} \int_{Q_{\Omega}} \frac{|u(x)-u(y)|^{2}}{|x-y|^{n+2 s}} d x d y \\
& +b_{\varepsilon} \int_{\Omega} W(u(x)) d x,
\end{aligned}
$$

where

$$
a_{\varepsilon}:= \begin{cases}\varepsilon^{2 s-1} & \text { if } s \in(1 / 2,1) \\ |\log \varepsilon|^{-1} & \text { if } s=1 / 2 \\ 1 & \text { if } s \in(0,1 / 2)\end{cases}
$$

and

$$
b_{\varepsilon}:=\varepsilon^{-2 s} a_{\varepsilon}= \begin{cases}\varepsilon^{-1} & \text { if } s \in(1 / 2,1), \\ \varepsilon^{-1}|\log \varepsilon|^{-1} & \text { if } s=1 / 2, \\ \varepsilon^{-2 s} & \text { if } s \in(0,1 / 2) .\end{cases}
$$

The coefficients $a_{\varepsilon}$ and $b_{\varepsilon}$ are tuned with respect to the energy of a one-dimensional layer joining -1 and +1 , and, in this way, the functional $\varepsilon_{\varepsilon}$ in (28) satisfies some interesting $\Gamma$-convergence properties. Remarkably, these properties are very sensitive to the fractional parameter $s$ : indeed, when $s<1 / 2$ the $\Gamma$-convergence theory obtains the $\sigma$-perimeter introduced in (6), with $\sigma:=2 s$, but when $s \geq$ $1 / 2$, the nonlocal character of the problem is lost in the limit, and the $\Gamma$-convergence theory boils down to the classical one in (20), (21), and (22).
More precisely, one can define

$$
\varepsilon_{0}(u ; \Omega)=\varepsilon_{0}(u):=\left\{\begin{array}{lc}
c \operatorname{Per}(E, \Omega) & \text { if } s \in[1 / 2,1) \\
& \text { and } u=\Xi_{E} \\
& \text { for some set } E, \\
& \text { if } s \in(0,1 / 2) \\
c \operatorname{Per}_{2 s}(E, \Omega) & \text { and } u=\Xi_{E} \\
& \text { for some set } E, \\
& \text { otherwise. }
\end{array}\right.
$$

In this sense, the functional $\varepsilon_{0}$ is the "natural replacement" of the one in (22) to deal with the $\Gamma$-convergence of nonlocal phase transitions, in the sense that the statements in (20), (21), and (22) hold true in this case with $\mathcal{F}_{\varepsilon}$ replaced by $\mathcal{E}_{\varepsilon}$ and $\mathcal{F}_{0}$ replaced by $\varepsilon_{0}$.

The convergence of level sets in (23) has also a perfect counterpart for the minimizers of $\mathcal{E}$, as established in [SV14]. These results also rely on suitable density estimates which guarantee that (25) also holds true for minimizers of $\mathcal{E}$.

However, in this case, an energy estimate as in (24) exhibits a significant difference, depending on the fractional exponent $s$. Indeed, in this framework, we have that if $R \geq 2$ and $u$ is a minimizer of $\mathcal{E}$ in $B_{R+1}$, then

$$
\mathcal{E}_{B_{R}}(u) \leq \begin{cases}C R^{n-1} & \text { if } s \in(1 / 2,1), \\ C R^{n-1} \log R & \text { if } s=1 / 2, \\ C R^{n-2 s} & \text { if } s \in(0,1 / 2),\end{cases}
$$

for some $C>0$.

In spite of its quantitative difference with (24), the estimate in (29) is sufficient to determine that the interface contribution is "negligible" with respect to the Lebesgue measure of large balls. Moreover, such an estimate is in agreement with the energy of one-dimensional layers. 
We refer to Table 2 for a schematic representation of similarities and differences of classical and nonlocal phase transitions in terms of $\Gamma$-limits and density estimates.

\section{Geometric Flows}

Another classical topic in geometric analysis is the study of evolution equations describing the motion of set boundaries. In this framework, at any time $t$, one prescribes the speed $V$ of a hypersurface $\partial E_{t}$ in the direction of the outer normal to $E_{t}$.

In this section, in light of the setting in (7), we will describe analogies and differences between the classical and the nonlocal mean curvature flows.

6.1. Mean curvature flow. One of the widest problems in geometric analysis focuses on the case in which the normal velocity $V$ of the flow is taken to be the classical mean curvature. This flow decreases the classical perimeter and has a number of remarkable properties. A convenient description of the mean curvature flow can be given in terms of viscosity solutions, using a level set approach, as presented by L. C. Evans and J. Spruck in 1991. If the initial hypersurface satisfies a Lipschitz condition, then the mean curvature flow possesses a short time existence theory, as proved by K. Ecker and G. Huisken in 1991.

In general, the flow may develop singularities, and the understanding of the different possible singularities is a very rich and interesting field of investigation in itself.

The planar case $n=2$ presents some special features. In this case, the flow becomes "curve shortening" in the sense that its special characteristic is to decrease the length of a given closed curve as fast as possible. In this setting, it was proved by M. A. Grayson in 1987 that the curve-shortening flow makes every smooth closed embedded curve in the plane shrink smoothly to round points (i.e., the curve shrinks, becoming "closer and closer to a circle"). More precisely: on the one hand, as shown by $\mathrm{M}$. Gage and $\mathrm{R}$. S. Hamilton in 1986, if the initial curve is convex, then the evolving curve remains convex and becomes asymptotically circular as it shrinks; on the other hand, as shown by M. A. Grayson in 1987, if the curve is only embedded, then no singularity develops before the curve becomes convex, then shrinking to a round point.

These results can also be interpreted in light of a "distance comparison property." Namely, given two points $p$ and $q$ on the evolving surface, one can consider the "extrinsic" distance $d=d(p, q)$, given by the Euclidean distance of $p$ and $q$, and the arc-length "intrinsic" distance $\ell=$ $\ell(p, q)$, which measures the distance of $p$ and $q$ along the curve. For a closed curve of length $L$, one can also define

$$
\lambda:=\frac{L}{\pi} \sin \frac{\pi \ell}{L} .
$$

Then, as proved by G. Huisken in 1998, we have that the minimal ratio $\rho:=\min \frac{d}{\lambda}$ is nondecreasing under the curve shortening flow (and, in fact, strictly increasing unless the curve is a circle). In this spirit, the ratio $\rho$ plays the role of an improving isoperimetric quantity which measures the deviation of the evolving curve from a round circle. In particular, if a curve developed a "neckpinch," it would present two points for which $d=0$ and $\ell>$ 0 ; hence $\rho=0$. As a consequence, the monotonicity proved by G. Huisken in 1998 immediately implies that planar curves do not develop neckpinches under the curveshortening flow. More generally, the results proved by G. Huisken in 1998 can be seen as a quantitative refinement of those by M. Gage and R. S. Hamilton in 1986 and M. A. Grayson in 1987.

In any case, for the mean curvature flow, the formation of neckpinch singularity for the evolving hypersurface can only occur (and it occurs) in dimension $n \geq 3$, as established by G. Huisken in 1990.

6.2. Nonlocal mean curvature flow. A natural variant of the flow discussed in section 6.1 consists of taking as normal velocity the nonlocal mean curvature of the evolving set, as given in (7). The main feature of this evolution problem is that it decreases the nonlocal perimeter defined in (6).

A detailed viscosity solution theory for the nonlocal mean curvature flow has been presented by A. Chambolle, M. Morini, and M. Ponsiglione in 2015.

In the viscosity setting, given an initial set $E \subset \mathbb{R}^{n}$, the evolution of $E$ is described by level sets of a function $u_{E}=$ $u_{E}(x, t)$ which solves a parabolic problem driven by the nonlocal mean curvature. More precisely, the evolution of $E$ is trapped between an outer and an inner flow defined by

$$
\begin{array}{ll}
E^{+}(t):=\left\{x \in \mathbb{R}^{n} \text { s.t. } u_{E}(x, t) \geq 0\right\} \\
\text { and } \quad & E^{-}(t):=\left\{x \in \mathbb{R}^{n} \text { s.t. } u_{E}(x, t)>0\right\} .
\end{array}
$$

The ideal case would be the one in which these two flows coincide "up to a smooth manifold" which describes the evolving surface, i.e., the case in which

$$
\begin{aligned}
\Sigma_{E}(t) & :=E^{+}(t) \backslash E^{-}(t) \\
& =\left\{x \in \mathbb{R}^{n} \text { s.t. } u_{E}(x, t)=0\right\}
\end{aligned}
$$

is a nice hypersurface, but, in general, such a property is not warranted by the notion of viscosity solutions.

A short-time existence theory of smooth solutions of the nonlocal mean curvature flow has been recently established by V. Julin and D. La Manna, under the assumption that the initial hypersurface is of class $C^{1,1}$. As far as we know, it is still an open problem to determine whether the nonlocal mean curvature flow possesses a short-time existence theory of smooth solutions for Lipschitz initial data (this would provide a complete counterpart of the classical results by K. Ecker and G. Huisken in 1991). 


\begin{tabular}{|l|l|l|l|}
\hline & $\begin{array}{l}\text { phase transitions } S \in \\
(0,1 / 2)\end{array}$ & phase transitions $s=1 / 2$ & $\begin{array}{l}\text { phase transitions } s \quad \in \\
(1 / 2,1]\end{array}$ \\
\hline$\Gamma$-limit & nonlocal perimeter & classical perimeter & classical perimeter \\
\hline energy estimates & $R^{n-2 S}$ & $R^{n-1} \log R$ & $R^{n-1}$ \\
\hline
\end{tabular}

Table 2. Classical versus nonlocal phase transitions.

It is certainly interesting to detect suitable information that is preserved by the nonlocal mean curvature flow. For instance: the fact that the nonlocal mean curvature flow preserves the positivity of the nonlocal mean curvature itself was established by M. Sáez and E. Valdinoci in 2019, and the preservation of convexity was proved by A. Chambolle, M. Novaga, and B. Ruffini in 2017.

The structure of the nonlocal mean curvature flow happens to be very different from the classical case, even in the planar situation: for example, the classical shrinking result by M. A. Grayson in 1987 showing that planar curves shrink to a point without developing singularities does not carry over to the fractional case, as proved in [CSV18], where a neckpinch singularity in the plane was exhibited.

As a matter of fact, in [CSV18] it was established that for the nonlocal mean curvature flow, the formation of neckpinch singularity for the evolving hypersurface occurs in dimension $n \geq 2$, thus providing a nonlocal counterpart of the result by G. Huisken in 1990 .

Another important difference between the classical and the nonlocal mean curvature flows is the "fattening phenomena" of viscosity solutions, in which the set in (30) may develop a nonempty interior (thus failing to be a nice hypersurface), as investigated in [CDNV19]. For instance, one can consider the case in which the initial set is the "cross in the plane" given by

$$
E=\left\{(x, y) \in \mathbb{R}^{2} \text { s.t. }|x|>|y|\right\} .
$$

In this situation, using the notation in (30), one can prove that $\Sigma_{E}(t)$ is nontrivial and immediately develops a positive measure: more precisely, we have that $\Sigma_{E}(t)$ contains the ball $B_{c t} \frac{1}{1+s}$ for some $c>0$.

On the one hand, this result is in agreement with the classical case, since also the classical mean curvature flow develops this kind of situation when starting from the set in (31) (see the 1991 article by L. C. Evans and J. Spruck).

On the other hand, the fattening phenomena for the nonlocal situation offers a number of surprises with respect to the classical case (see [CDNV19]). First of all, the quantitative properties of the interaction kernels play a decisive role in the development of the fat portions of $\Sigma_{E}(t)$ and in general in the evolution of the set $E$. For instance, if the interaction kernel $\frac{1}{|x-y|^{n+\sigma}}$ in (3) is replaced by a different kernel that is smooth and compactly supported, then the nonlocal mean curvature flow of the cross $E$ in (31) does not develop any fattening and, more precisely, the evolution of $E$ would be $E$ itself (in a sense, we switch from a fattening situation, produced by singular kernels with slow decay, to a "pinning effect" produced by smooth and compactly supported kernels).

Furthermore, there are cases in which the fattening phenomena are different according to the type of local or nonlocal mean curvature flow that we consider (see the 1991 article by L. C. Evans and J. Spruck). For instance, if the initial set is made by two tangent balls in the plane such as

$$
E:=B_{1}(-1,0) \cup B_{1}(1,0),
$$

then for the nonlocal mean curvature flow, using the notation in (30), we have that $\Sigma_{E}(t)$ has empty interior for all $t>0$. This situation is different with respect to the classical mean curvature flow, which immediately develops fattening (as discussed by G. Bellettini and M. Paolini in 1994).

The nonlocal mean curvature flow (and its many variants) is a great source of intriguing questions and mathematical adventures. Among the interesting questions remaining open, we mention that it is not known whether the nonlocal mean curvature flow possesses a nonlocal version of the monotonicity formula obtained by G. Huisken in 1998, or what a natural replacement of it could be. This question is also related to a deeper understanding of the singularities of the nonlocal mean curvature flow.

For the sake of shortness and to comply with the journal's settings, we deferred a more comprehensive list of references to the arXiv version of this note: https: //arxiv .org/abs/2003.13234.

ACKNOWLEDGMENTS. The author is a member of INdAM/GNAMPA and AustMS. Her research is supported by the Australian Research Council Discovery Project DP170104880, "N.E.W. Nonlocal Equations at Work," and by the DECRA Project DE180100957, "PDEs, free boundaries and applications."

It is a pleasure to thank Enrico Valdinoci for his comments on a preliminary version of this manuscript.

\section{References}

[BFV14] Begoña Barrios, Alessio Figalli, and Enrico Valdinoci, Bootstrap regularity for integro-differential operators and its application to nonlocal minimal surfaces, Ann. Sc. Norm. Super. Pisa Cl. Sci. (5) 13 (2014), no. 3, 609-639. MR3331523 
[CC19] Xavier Cabré and Matteo Cozzi, A gradient estimate for nonlocal minimal graphs, Duke Math. J. 168 (2019), no. 5, 775-848, DOI 10.1215/00127094-20180052. MR3934589

[CDDP16] Eleonora Cinti, Juan Davila, and Manuel Del Pino, Solutions of the fractional Allen-Cahn equation which are invariant under screw motion, J. Lond. Math. Soc. (2) 94 (2016), no. 1, 295-313, DOI 10.1112/jlms/jdw033. MR3532174

[CDNV19] Annalisa Cesaroni, Serena Dipierro, Matteo Novaga, and Enrico Valdinoci, Fattening and nonfattening phenomena for planar nonlocal curvature flows, Math. Ann. 375 (2019), no. 1-2, 687-736, DOI 10.1007/s00208-018-17936. MR4000255

[CDSS16] L. Caffarelli, D. De Silva, and O. Savin, Obstacletype problems for minimal surfaces, Comm. Partial Differential Equations 41 (2016), no. 8, 1303-1323, DOI 10.1080/03605302.2016.1192646. MR3532394

[CRS10] L. Caffarelli, J.-M. Roquejoffre, and O. Savin, Nonlocal minimal surfaces, Comm. Pure Appl. Math. 63 (2010), no. 9, 1111-1144, DOI 10.1002/cpa.20331. MR2675483

[CSV18] Eleonora Cinti, Carlo Sinestrari, and Enrico Valdinoci, Neckpinch singularities in fractional mean curvature flows, Proc. Amer. Math. Soc. 146 (2018), no. 6, 2637-2646, DOI 10.1090/proc/14002. MR3778164

[CSV19] Eleonora Cinti, Joaquim Serra, and Enrico Valdinoci, Quantitative flatness results and BV-estimates for stable nonlocal minimal surfaces, J. Differential Geom. 112 (2019), no. 3, 447-504, DOI 10.4310/jdg/1563242471. MR3981295

[CV13] Luis Caffarelli and Enrico Valdinoci, Regularity properties of nonlocal minimal surfaces via limiting arguments, Adv. Math. 248 (2013), 843-871, DOI 10.1016/j.aim.2013.08.007. MR3107529

[DdPW18] Juan Dávila, Manuel del Pino, and Juncheng Wei, Nonlocal s-minimal surfaces and Lawson cones, J. Differential Geom. 109 (2018), no. 1, 111-175. MR3798717

[DFPV13] Serena Dipierro, Alessio Figalli, Giampiero Palatucci, and Enrico Valdinoci, Asymptotics of the $s$ perimeter as $s \searrow 0$, Discrete Contin. Dyn. Syst. 33 (2013), no. 7, 2777-2790, DOI 10.3934/dcds.2013.33.2777. MR3007726

[DSV16] Serena Dipierro, Ovidiu Savin, and Enrico Valdinoci, Graph properties for nonlocal minimal surfaces, Calc. Var. Partial Differential Equations 55 (2016), no. 4, Art. 86, 25, DOI 10.1007/s00526-016-1020-9. MR3516886

[DSV17] Serena Dipierro, Ovidiu Savin, and Enrico Valdinoci, Boundary behavior of nonlocal minimal surfaces, J. Funct. Anal. 272 (2017), no. 5, 1791-1851, DOI 10.1016/j.jfa.2016.11.016. MR3596708

[DSV] Serena Dipierro, Ovidiu Savin, and Enrico Valdinoci, Boundary properties of fractional objects: flexibility of linear equations and rigidity of minimal graphs, J. Reine Angew. Math., to appear, arXiv:1907.01498, 2019.

[DSV20] Serena Dipierro, Ovidiu Savin, and Enrico Valdinoci, Nonlocal minimal graphs in the plane are generically sticky, Comm. Math. Phys. 376 (2020), no. 3, 2005-2063, DOI 10.1007/s00220-020-037718. MR4104542

[FV17] Alessio Figalli and Enrico Valdinoci, Regularity and Bernstein-type results for nonlocal minimal surfaces, J. Reine Angew. Math. 729 (2017), 263-273, DOI 10.1515/crelle2015-0006. MR3680376

[ROS14] Xavier Ros-Oton and Joaquim Serra, The Dirichlet problem for the fractional Laplacian: regularity up to the boundary, J. Math. Pures Appl. (9) 101 (2014), no. 3, 275-302. MR3168912

[SV12] Ovidiu Savin and Enrico Valdinoci, $\Gamma$-convergence for nonlocal phase transitions, Ann. Inst. H. Poincaré Anal. Non Linéaire 29 (2012), no. 4, 479-500. MR2948285

[SV13] Ovidiu Savin and Enrico Valdinoci, Regularity of nonlocal minimal cones in dimension 2, Calc. Var. Partial Differential Equations 48 (2013), no. 1-2, 33-39, DOI 10.1007/s00526-012-0539-7. MR3090533

[SV14] Ovidiu Savin and Enrico Valdinoci, Density estimates for a variational model driven by the Gagliardo norm, I. Math. Pures Appl. (9) 101 (2014), no. 1, 1-26. MR3133422

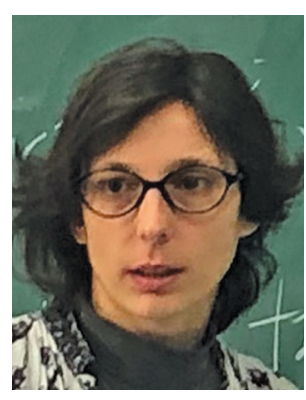

Serena Dipierro

Credits

Opening image is courtesy of Eureka_89 via Getty. Figures 1-3 are courtesy of Serena Dipierro.

Photo of Serena Dipierro is courtesy of Ruofei Yao. 